\title{
Synthesis and Characterization of Styrene-Butadiene Block Copolymer/Silicate Hybrid Materials via the Sol-Gel Process I. Four-Arm Star Styrene-Butadiene Block Copolymer/Silica Hybrids
}

\author{
Weibin CHEN, Hanqiao Feng, ${ }^{\dagger}$ and Chaohui Ye \\ Laboratory of Magnetic Resonance and Atomic Molecular Physics, Wuhan Institute of Physics and Mathematics, \\ Chinese Academy of Sciences, Wuhan 430071, People's Republic of China
}

(Received April 10, 1997)

\begin{abstract}
Novel hybrid materials incorporating four-arm star styrene-butadiene block copolymer (SB-4A) with tetraethoxysilane (TEOS) have been successfully prepared by a sol-gel process. The properties, microstructure, and dynamics of the hybrid materials were investigated in detail by means of dynamic mechanical analysis (DMA), thermogravimetry (TG), and NMR techniques. The results indicate that the properties of the hybrids strongly depend on the TOES content and the silica networks prefer to form in the polystyrene (PS) domains. There exists an optimum range of TEOS content, in this work $30-50 \%$ by weight, within which the properties of the hybrids obtained were well improved: storage modulus increases, thermal stability raises, and the high-temperature $T_{\mathrm{g}}$ shifts to higher temperature. ${ }^{29} \mathrm{Si}$ NMR spectrum shows that in the hybrids with well improved properties silica networks formed with $Q^{4}$ in the greatest abundance.

KEY WORDS Four-Arm Star Styrene-Butadiene / Tetraethoxysilane Hybrid Materials / Microstructure / Mobility / Solid-State ${ }^{13} \mathrm{C}$ and ${ }^{29} \mathrm{Si}$ Nuclear Magnetic Resonance / Dynamic Mechanical Analysis /
\end{abstract}

Elastomers usually used as composite materials in industrial products. Inorganic fillers, such as carbon black or reinforcing silica are added. The fillers make an elastomer stronger and are of great importance from the practical point of view. Usually, fillers are mechanically introduced into a polymer by milling. ${ }^{1,2}$ This slow, energy-intensive process may cause chemical degradation of the polymer chains. Besides, undesirable particulate agglomerates often remain even after aggressive milling. Small, separated particles are ideal for reinforcing a polymer matrix. It is, therefore, desirable to explore alternate methods of incorporating an inorganic filler into an elastomer.

The sol-gel process, with its associated mild conditions, offers a new approach to the synthesis of composite materials with domain sizes approaching the molecular level when conducted in polymeric media. ${ }^{3-6}$

Typical hydrolysis-condensation reactions for tetraethoxysilane (TEOS) are shown below.

$$
\begin{aligned}
& \text { Hydrolysis: } \mathrm{Si}\left(\mathrm{OE}_{\mathrm{t}}\right)_{4}+n \mathrm{H}_{2} \mathrm{O} \rightarrow \\
& \mathrm{Si}\left(\mathrm{OE}_{\mathrm{t}}\right)_{4-n}(\mathrm{OH})_{n}+n \mathrm{E}_{\mathrm{t}} \mathrm{OH}
\end{aligned}
$$

Condensation (alcohol-producing):

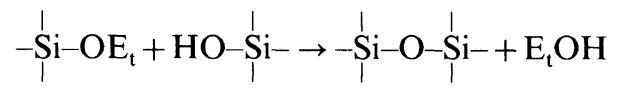

Condensation (water-producing):

$$
\stackrel{+}{\mathrm{S}} \mathrm{i}-\mathrm{OH}+\mathrm{HO}-\underset{1}{\mathrm{~S} i}-\rightarrow-{ }_{1}^{\mathrm{S}} \mathrm{i}-\mathrm{O}-\underset{1}{\mathrm{~S} i}--+\mathrm{H}_{2} \mathrm{O}
$$

The most direct route to the formation of organicinorganic composite materials through the sol-gel process involves carrying out the inorganic hydrolysis and condensation reactions in the presence of a preformed organic polymer. In this work, we selected four-

\footnotetext{
† To whom correspondence should be addressed.
}

arm star styrene-butadiene block copolymer (SB-4A) as the performed polymer to conduct the sol-gel reactions with TEOS by the route mentioned above, anticipating to improve the properties of SB-4A by the formaton of $\mathrm{SB}-4 \mathrm{~A} / \mathrm{SiO}_{2}$ hybrid materials.

\section{EXPERIMENTAL}

TEOS was obtained from the Fluka Company of Japan without further purification. SB-4A was obtained from the Synthetic Rubber Factor of Baling Petro-Chemical Co., and purified before use. The characteristic data of SB-4A is listed in Table I. Tetrahydrofuran (THF), purified before use, was used as solvent to insure homogeneous solutions.

SB-4A (1 g) was dissolved in THF $(10 \mathrm{ml})$, then TEOS and deionized water with defined contents (see Table II) were added to the above solution. The resulting mixture was refluxed $8 \mathrm{~h}$ in the oil bath $\left(65-70^{\circ} \mathrm{C}\right)$ under stirring. After cooling, the clear liquid was cast onto glass plate and air dried under ambient conditions for 3 days. Specimens were further dried at $40^{\circ} \mathrm{C}$ under vacuum for $6 \mathrm{~h}$ prior to testing. Dynamic mechanical analysis (DMA) experiments were done on a dynamic viscoelastometer (Rheovibron DDV-II-EA) at a heating rate of $3^{\circ} \mathrm{C} \mathrm{min}^{-1}$ from -120 to $+140^{\circ} \mathrm{C}$. The vibration frequency was $3.5 \mathrm{~Hz}$. The specimens used were about $2.6 \times 0.44 \times$ $0.015 \mathrm{~cm}$.

The thermogravimetry analysis was performed on a Shimadzu DT-30B thermal analyzer under nitrogen flow at a heating rate of $15^{\circ} \mathrm{Cmin}^{-1}$.

Solid state ${ }^{13} \mathrm{C}$ CPMAS and ${ }^{29} \mathrm{Si}$ MAS NMR experiments were performed on a Bruker MSL- $400 \mathrm{MHz}$ NMR spectrometer at $298 \mathrm{~K}$. The carbon- 13 resonance frequency was $100.63 \mathrm{MHz}$, and proton resonance frequency was $400.13 \mathrm{MHz}$. Dipolar decoupling field was about $49 \mathrm{kHz} .{ }^{13} \mathrm{C}$ spectra were referred to the shifts of methyl group carbons of hexamethyl benzene which was 
Table I. Characterization data of SB- $4 A^{a}$

\begin{tabular}{ccc}
\hline$M_{n}{ }^{\mathrm{b}}$ & $\frac{\mathrm{SB}^{\mathrm{c}}}{\mathrm{wt} / \mathrm{wt}}$ & $\frac{\mathrm{PS} M_{n}}{\times 10^{-4}}$ \\
\cline { 3 - 3 } & $45 / 55$ & $\times 10^{-4}$ \\
\hline 15.43 & 453 \\
\hline
\end{tabular}

${ }^{a}$ PS blocks locate at the outer ends of the copolymer chains. ${ }^{b}$ Determined by membrane osmometry in toluene at $37^{\circ} \mathrm{C}$. ${ }^{\mathrm{c}}$ Determined by ${ }^{13} \mathrm{C}$ NMR.

Table II. Initial reactive multicomponent solutions

\begin{tabular}{|c|c|c|c|c|}
\hline \multirow{2}{*}{ Sample no. } & TEOS & SB-4A & $\mathrm{H}_{2} \mathrm{O}:$ TEOS & \multirow{2}{*}{$\begin{array}{c}\mathrm{SiO}_{2} \mathrm{wt}^{\%} \text { in } \\
\text { the final } \\
\text { products }^{\mathrm{a}}\end{array}$} \\
\hline & $\mathrm{m}$ & $\mathrm{g}$ & $\mathrm{mol} / \mathrm{mol}$ & \\
\hline 1 & 0 & 1 & - & - \\
\hline 2 & 0.4 & 1 & $4: 1$ & 9.9 \\
\hline 3 & 0.6 & 1 & $4: 1$ & 13.8 \\
\hline 4 & 1.0 & 1 & $4: 1$ & 21.2 \\
\hline 5 & 1.6 & 1 & $4: 1$ & 30.1 \\
\hline
\end{tabular}

${ }^{a}$ Assuming the reaction is complete.

$16.9 \mathrm{ppm} .{ }^{29} \mathrm{Si}$ resonance frequency was $79.46 \mathrm{MHz}$, and MAS rate was $1.5 \mathrm{kHz}$. The ${ }^{29} \mathrm{Si}$ spectrum was referred to the chemical shift of methyl group silicons of tetrakis(trimethylsilyl)silane (TKS) which is $-9.8 \mathrm{ppm}$.

\section{RESULTS AND DISCUSSION}

In this study, the amount of water added to initiate the hydrolysis reaction was in stoichiometry. We found that the catalytic conditions have a dramatic effect on the structure of the inorganic network formed. The final films showed distinct phase separation when acid $(\mathrm{HCl})$ or base $\left(\mathrm{NH}_{3} \mathrm{H}_{2} \mathrm{O}\right)$ were used as catalyst. However, under neutral conditions, the same as pure SB-4A film, the final $\mathrm{SB}-4 \mathrm{~A} / \mathrm{SiO}_{2}$ hybrid materials are smooth and semitransparent films except sample 5 whose film shows relatively poorer transparency.

Shown in Figure 1 are plots of storage modulus $\left(E^{\prime}\right)$ and $\tan \delta v s$. temperature of SB-4A and its hybrids with different TEOS content, respectively. The glass transition temperatures $\left(T_{\mathrm{g}}\right)$, obtained from their $\tan \delta$ curves, of SB-4A and its hybrids are listed in Table III. There are several features deserve to be mentioned about Figure 1: (1) for pure SB-4A, two $T_{\mathrm{g}}$ values, -86 and $+93^{\circ} \mathrm{C}$, are observed, which can be assigned to the $T_{\mathrm{g}} \mathrm{s}$ of polybutadiene (PBD) and polystyrene (PS) segments, respectively. 2) the $T_{\mathrm{g}}$ values both $\mathrm{PBD}$ and PS blocks in hybrids show quite different dependence on the TEOS content. For PS block in hybrids, its $T_{\mathrm{g}}$ firstly increases with the TEOS content until a maximum $T_{\mathrm{g}}$ reaches. Then further increase in TEOS content will result in a slight decrease in $T_{\mathrm{g}}$ of PS block. Of course, its $T_{\mathrm{g}}$ is still higher than that of PS block in pure SB-4A. Interestingly, it seems that no PBD block in all the hybrids 'feels' the existence of silica networks since its $T_{\mathrm{g}}$ value remains unchanged with the increase of TEOS content. 3) it is clear to see that the addition of TEOS gives rise to an obvious effect on the storage modulus of hybrids. There exists an optimum range of TEOS content, in this case $30-50 \%$ by weight, within which the hybrids formed,
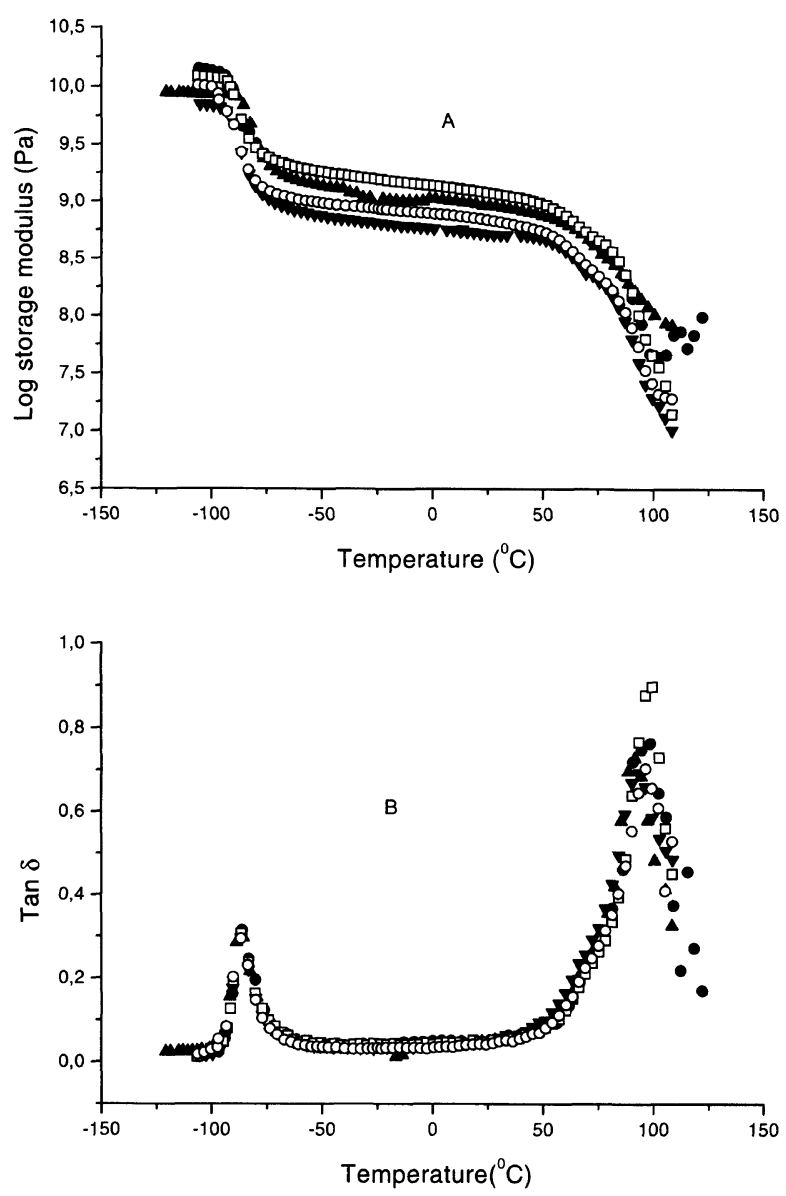

Figure 1. DMA curves of SB-4A and its hybrids for: (A) pure SB$4 A$, ( ) sample $2,(\bigcirc)$ sample $3,(\square)$ sample 4 , and $(\bigcirc)$ sample 5.

Table III. $T_{\mathrm{g}}$ 's from $\tan \delta$ of SB-4A and its hybrids

\begin{tabular}{ccc}
\hline Sample no. & $T_{\mathbf{g}}{ }^{1} /{ }^{\circ} \mathrm{C}$ & $T_{\mathbf{g}}{ }^{2} /{ }^{\circ} \mathrm{C}$ \\
\hline 1 & -86 & +93 \\
2 & -87 & +96 \\
3 & -86 & +98 \\
4 & -87 & +99 \\
5 & -87 & +96 \\
\hline
\end{tabular}

samples 3 and 4, exhibit well improved properties: their storage modulus increase as compared with that of pure SB-4A, and the increased PS $T_{\mathrm{g}}$ is the highest. When the TEOS content in hybrids is out of the range, samples 2 and 5 , the storage modulus of hybrids is lower than that of pure SB-4A. The complicated dependence of hybrid modulus on TEOS content suggests that the microstructures of the hybrids formed are quite different. Without doubt, more perfect silica networks, homogeneously dispersed in PS domains, are responsible for the well improved properties of samples 3 and 4 . For sample 2 , the TEOS content added is too smaller to form silica networks big enough to incorporate all the PS blocks in spite of homogeneous dispersion. Thus, the weaker interaction between rich PS region and rich silica particles or networks region may result in the decreased storage modulus. For sample 5 with the highest TEOS content, the agglomeration of silica may be the main cause of the lowered modulus. As mentioned before, the transparency of film of sample 5 is poorer than that of pure SB-4A's. 


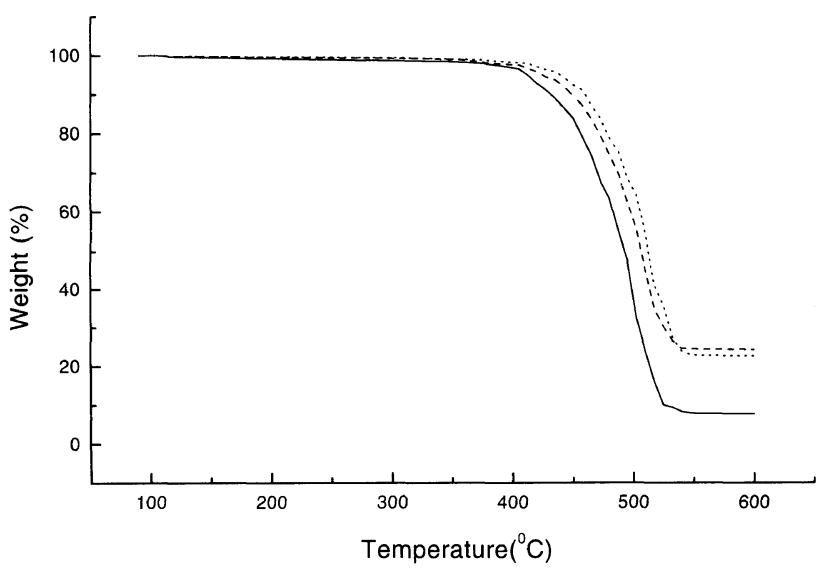

Figure 2. Thermogravimetry curves for: ( - ) pure SB-4A, (--.--) sample 4 , and (---) sample 5 .

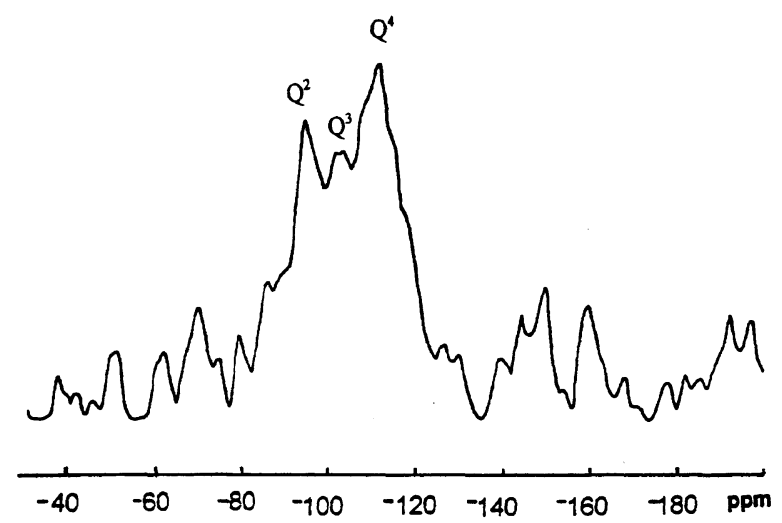

Figure 3. ${ }^{29} \mathrm{Si}$ solid-state MAS NMR spectrum of sample 4 .

As expected, the thermal stability of hybrids also shows an apparent dependence on the microstructure of hybrids. Figure 2 gives the thermogravimetry (TG) curves for pure SB-4A and its hybrids. It is found that the start temperature of decomposition for hybrids is $40^{\circ} \mathrm{C}$ higher than that of pure SB-4A, and the sample 4 with higher storage modulus exhibits higher thermal stability than sample 5 with lower storage modulus, although the latter contains more TEOS than the former. Another point worthy to be mentioned is that, as expected, the quantity of residue in sample 5 is more than that of sample 4 , indicating that there is more $\mathrm{SiO}_{2}$ formed in sample 5 . However, the quantity of residue in sample 5 is only $17 \%$ by weight, which is much less than the theoretic value $(30.1 \mathrm{wt} \%)$ with an assumption of complete reaction (see Table II). A quite different situation occurs for sample 4 , the quantity of residue is $15.5 \%$ by weight, which is close to the theoretic value $(21.2 \mathrm{wt} \%)$. The above results clearly indicate that the silica networks formed in sample 4 are more perfect than those in sample 5, which play a key role in determining the storage modulus, glass transition temperature and thermal stability of hybrids.

The conclusion that more perfect silica networks formed in sample 4 is further supported by the ${ }^{29} \mathrm{Si}$ MAS NMR spectrum shown in Figure 3. Based on peak assignment from model compounds, ${ }^{7,8}$ three states of Si coordination about $\mathrm{SiO}_{4}$ tetrahedra- $Q^{2}, Q^{3}$, and $Q^{4}$, are evident with $Q^{4}$ in the greatest abundance. Due

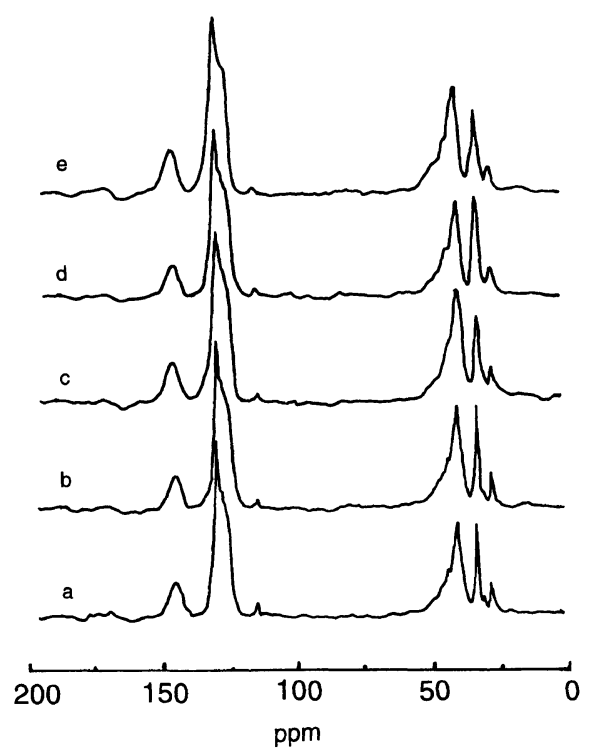

Figure 4. ${ }^{13} \mathrm{C}$ solid-state CPMAS NMR spectra of SB-4A and its hybrids for: (a) pure SB-4A, (b) sample 2, (c) sample 3, (d) sample 4, and (e) sample 5 .

to insufficient condensation or hydrolysis, some $\mathrm{SiO}_{4}$ groups are di- and tri-substituted. No $Q^{1}$ or TEOS peak appear. Meanwhile, the ${ }^{13} \mathrm{C}$ CPMAS NMR spectra of pure SB-4A and its hybrids, shown in Figure 4, clearly indicate that hydrolysis of TEOS in all the hybrids is complete since there is no resonance peak from ethoxy groups, which, if exist, should appear around $20 \mathrm{ppm}$ and $70 \mathrm{ppm}$. As consequence, there exist a great quantity of silanol in the hybrids because each $Q^{3}$ and $Q^{2}$ contains one and two silanol groups, respectively.

The results mentioned above suggest that the restrictions of silica networks imposed on PBD and PS blocks are rather different. It seems that silica networks prefer to form in PS domains and a few silica network formed in PBD domains. As a result, higher restriction should be imposed on the molecular movement of PS blocks in the hybrids with well improved properties, like samples 3 and 4, and lower restriction will be imposed on the PBD chains or PS chains in the samples 2 and 5. Therefore, as a powerful tool for characterizing microstructure and mobility of polymers, NMR spin-relaxation is expected to offer more detailed information about them. ${ }^{13} \mathrm{C}$ spin-lattice relaxation time in the rotating frame, $T_{1 \rho}(C)$, is sensitive to the molecular motion in the range of mid-kilohertz, which is particular interest in the case of polymers because the mechanical properties are also governed by the molecular dynamics in this frequency range. ${ }^{9-11}$ Although the measured $T_{1 \rho}^{*}(C)$ may contain the spin-spin contribution $T_{\mathrm{CH}}^{\mathrm{D}}$.

$$
1 / T_{1 \rho}^{*}(C)=1 / T_{1 \rho}(C)+1 / T_{\mathrm{CH}}^{\mathrm{D}}
$$

it is reasonable to explain $T_{1 \rho}^{*}(C)$ data of amorphous polymers by molecular dynamics since in amorphous polymers the measured $T_{1 \rho}^{*}(C)$ is predominantly $(\approx 80 \%)$ determined by spin-lattice process. ${ }^{12}$

Shown in Figures 5(A) and (B) are the plots of logarithmic ${ }^{13} \mathrm{C}$ resonance intensity vs. ${ }^{13} \mathrm{C}$ spin-locking time for PBD chains (peak at $130 \mathrm{ppm}$ ) and PS chains (peak at $40 \mathrm{ppm}$ ), respectively, in SB-4A and its hybrids. 
Synthesis and Characterization of Organic-Inorganic Hybrids
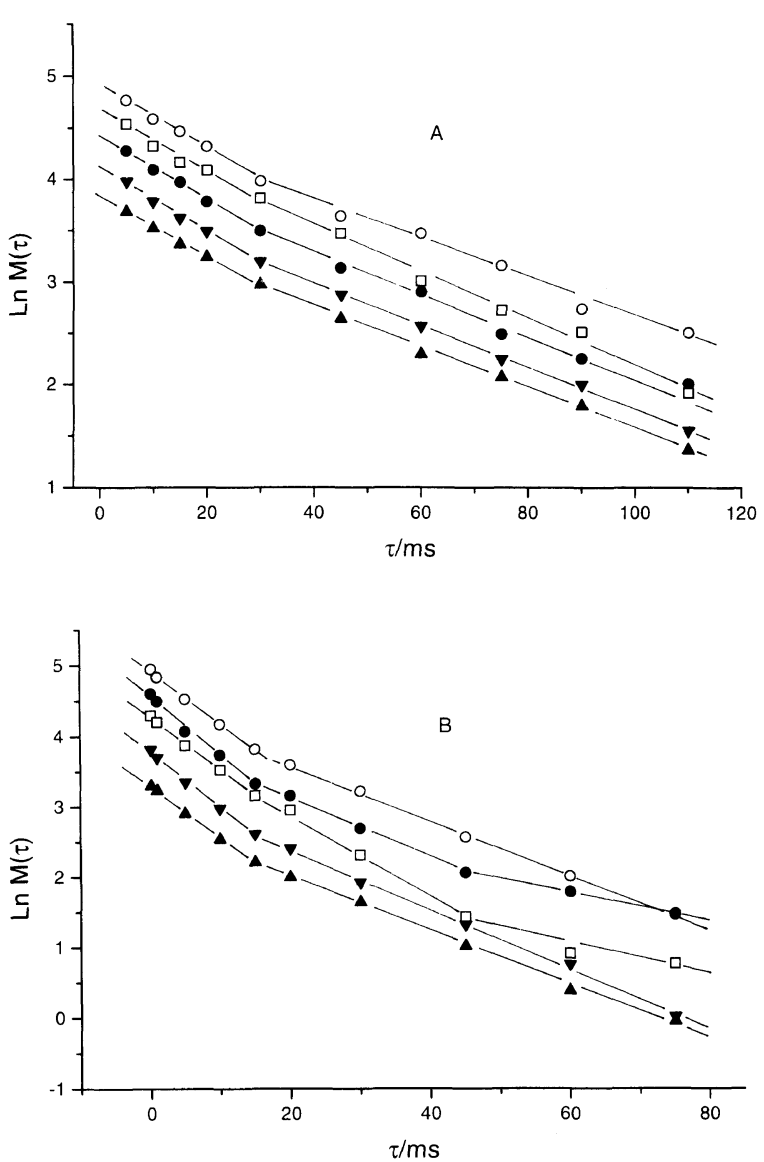

Figure 5. Plots of logarithmic ${ }^{13} \mathrm{C}$ resonance intensity vs. ${ }^{13} \mathrm{C}$ spin-locking time for: $(\boldsymbol{A})$ pure SB-4A, $(\boldsymbol{\nabla})$ sample $2,(\bullet)$ sample 3 , ( $\square$ ) sample 4, and (O) sample 5. A, PBD at $130 \mathrm{ppm}$; B, PS at $40 \mathrm{ppm}$.

The corresponding $T_{1 \rho}(C)$ values are listed in Table IV. From Figure 5 and Table IV it is clear to see that: 1) the decays of PBD and PS magnetization in pure SB-4A are all biexponential, this is in line with the fact that SB-4A is a microphase separation system. ${ }^{13}$ The biexponential decay of PBD or PS magnetization implies that there exist two kinds of PBD or PS domains with different molecular motions. Here, for PS blocks, the longer $T_{1 \rho}(C)$ can be assigned to the PS segments in PS rich phase and the shorter $T_{1 \rho}(C)$ to the PS blocks located in the interfacial region between PS domains and PBD domains. A similar situation also occurs for PBD blocks. 2) the addition of TEOS has quite different effects on the decays of PBD and PS magnetization. First, the addition of TEOS has almost no effect on the decay of PBD magnetization in all the hybrids, indicating that the molecular motion of PBD chains nearly remains unchanged. This is in excellent agreement with the DMA results that the $T_{\mathrm{g}} \mathrm{s}$ of $\mathrm{PBD}$ in all the hybrids are the same as that of PBD in pure SB-4A. Second, as expected, the decay of PS magnetization in hybrids with well improved properties is considerably changed by the addition of TEOS. A triexponential decay of PS magnetization in samples 3 and 4 are observed. Obviously a new region, in which the silica network imposes higher restriction on PS chains, forms due to the addition of silica networks. The restricted PS chains have a much longer $T_{1 \rho}(C)$ value. For samples 2 and 5 , it is also found that small effect of silica network on $T_{1 \rho}(C)$ of PS chains
Table IV. $T_{1 \rho}(C)$ values $(\mathrm{ms})^{\mathrm{a}}$ of SB-4A and its hybrids

\begin{tabular}{ccccccc}
\hline \multirow{2}{*}{$\begin{array}{c}\text { Sample } \\
\text { no. }\end{array}$} & \multicolumn{2}{c}{ PBD at $130 \mathrm{ppm}$} & & \multicolumn{3}{c}{ PS at $40 \mathrm{ppm}$} \\
\cline { 2 - 3 } \cline { 5 - 6 } & $T_{1 \rho^{(1)}}$ & $T_{1 \rho}{ }^{(2)}$ & & $T_{1 \rho}{ }^{(1)}$ & $T_{1 \rho}{ }^{(2)}$ & $T_{1 \rho}{ }^{(3)}$ \\
\hline 1 & 4.6 & 45.7 & & 6.9 & 24.0 & \\
2 & 5.3 & 44.8 & & 6.4 & 23.1 & \\
3 & 5.1 & 43.5 & & 5.9 & 23.1 & 49.1 \\
4 & 4.3 & 42.2 & & 6.4 & 20.1 & 46.6 \\
5 & 4.8 & 45.9 & & 4.8 & 30.0 & \\
\hline
\end{tabular}

${ }^{\mathrm{a}}$ Estimated error $\leq \pm 10 \%$

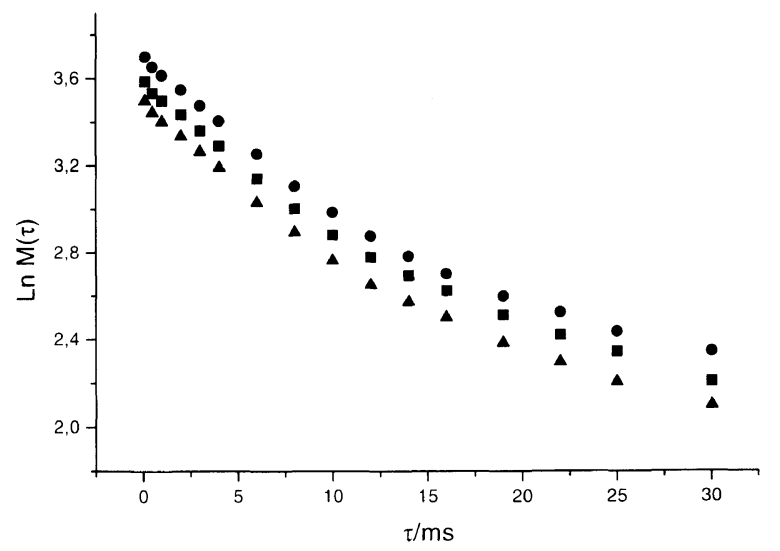

Figure 6. Plots of logarithmic ${ }^{1} \mathrm{H}$ resonance intensity $v s .{ }^{1} \mathrm{H}$ spinlocking time of PBD in: (ם) pure SB-4A, (A) sample 4, and sample 5 .

occurs.

To provide further evidence for the lack of silica networks in PBD domains, proton spin-lattice relaxation time in rotating frame, $T_{1 \rho}(H)$, was measured to study the homogeneity of PBD domains with and without TEOS. As well known, $T_{1 \rho}(H)$ has been used to characterize the miscibility or homogeneity of multicomponent polymer systems. ${ }^{14-16}$ It is expected that the existence of silica networks in PBD domains will change the $T_{1 \rho}(H)$ value of PBD to some degree. ${ }^{13}$ Shown in Figure 6 are the plots of logarithmic ${ }^{1} \mathrm{H}$ intensity $v s .{ }^{1} \mathrm{H}$ spin-locking time of PBD in pure SB-4A and its hybrids. Surprisingly, the decay of PBD magnetization in hybrids is exactly the same as that of PBD's in pure SB-4A. This again indicates that no detectable silica network exists in PBD domains, which, of course, is in good accordance with the results from DMA and $T_{1 \rho}(C)$ measurements. The reason why no silica network exists in PBD domains remains unknown and is currently under investigation. Finally, it should be pointed out that in the view of application it is applausible for silica networks to locate in PS domains since it does play the role of improving properties of material and has not effect on the elasticity of the material.

\section{CONCLUSION}

A novel hybrid material incorporating four-arm star styrene-butadiene block copolymer (SB-4A) with tetraethoxysilane (TEOS) has been successfully prepared by a sol-gel process. The TEOS content added has obvious effect on the properties of final hybrid materials. It was 
found that there exists an optimum range of TEOS content, in this case $30-50 \%$ by weight, within which the properties of hybrid materials obtained were well improved: storage modulus increases, thermal stability raises, and the high-temperature $T_{\mathrm{g}}$ shifts to higher temperature. Meanwhile, the low-temperature $T_{\mathrm{g}}$ remains unchanged. The results from DMA and NMR measurements indicate that silica networks prefer to form in PS domains, and few silica networks can be found in PBD domains. In the hybrid with well improved properties the silica networks formed with $Q^{4}$ in the greatest abundance.

Acknowledgment. The authors are grateful for the financial support granted by the National Natural Science Foundation of China and the Laboratory of Magnetic Resonance and Atomic and Molecular Physics, Wuhan Institute of Physics and Mathematics, Chinese Academy of Sciences.

\section{REFERENCES}

1. A. Kresztafkiewicz and M. Maik, Colloid and Polym. Sci., 265, 704 (1987).
2. O. E. Otterstedt, J.-E. A. Ottersted, J. Ekdahl, and J. Bachman, J. Appl. Polym. Sci., 34, 2575 (1987).

3. B. M. Novak, Adv. Mater., 5, 422 (1993).

4. H.-H. Huang, B. Orler, and G. L. Wilkes, Macromolecules, 20, $1322(1987)$.

5. H.-H. Huang and G. L. Wilkes, Polym. Bull., 18, 455 (1987).

6. H. Schmidt, J. Non-Cryst. Solids, 112, 419 (1989).

7. R. J. Hook, J. Non-Cryst. Solids, 1, 195 (1996).

8. W. G. Kimperer and S. D. Ramamurthi, Polym. Prepr., Am. Chem. Soc., Div. Polym. Chem., 28, 432 (1987).

9. V. D. Fedetov and H. Schneider, Ed., "Structure and Dynamics of Bulk Polymers by NMR Methods," VCH Publishers Inc., Weinheim, 1989.

10. J. Schaefer, E. O. Stejskal, and R. Buchdahl, Macromolecules, 10, 384 (1977)

11. J. Schaefer, E. O. Stejskal, T. R. Steger, M. D. Sefcik, and R. A. McKay, Macromolecules, 13, 1121 (1980).

12. R. Voelkel, Angew. Chem., 27, 1468 (1988).

13. H. Feng, Z. Feng, H. Yuan, and L. Shen, Macromolecules, 27, 7830 (1994).

14. E. O. Stejskal, J. Schaefer, M. D. Sefcik, and R. A. McKey, Macromolecules, 14, 275 (1981).

15. R. A. Komoroski, Ed., "High Resolution NMR Spectroscopy of Synthetic Polymers in Bulk," VCH Publishers, Inc., Weinheim, 1986.

16. H. Feng, C. Ye, P. Zhang, and Z. Feng, Macromol. Chem. Phys., 196, 2587 (1995). 